Pacific Journal of Mathematics

THE SLOW SHEARING MOTION OF A LIQUID PAST A 


\title{
THE SLOW SHEARING MOTION OF A LIQUID PAST A SEMI-INFINITE PLANE
}

\author{
G. Power and D. L. Scott-Hutton
}

The problem of slow shearing motion of liquid past a semi-infinite plane, which was first attempted by Dean [1], is treated in a rather more straightforward manner, and a different type of solution is found. The stream-function is biharmonic and vanishes, together with its normal derivative, at all points of the fluid boundary and must be such as to yield uniform shearing at a great distance from the boundary. It has not been found possible to satisfy all boundary conditions exactly, but a solution, involving an infinite number of arbitrary constants, is obtained which satisfies most of the necessary conditions. These arbitrary constants, here restricted to eight as a first approximation, are chosen to give the best possible result. Expressions for the stream-function and fluid pressure are obtained for specific regions, verifying known results including those for shear flow, for flow between parallel planes and for flow at a sharp corner. Finally, a plane elastic state analogy is pointed out.

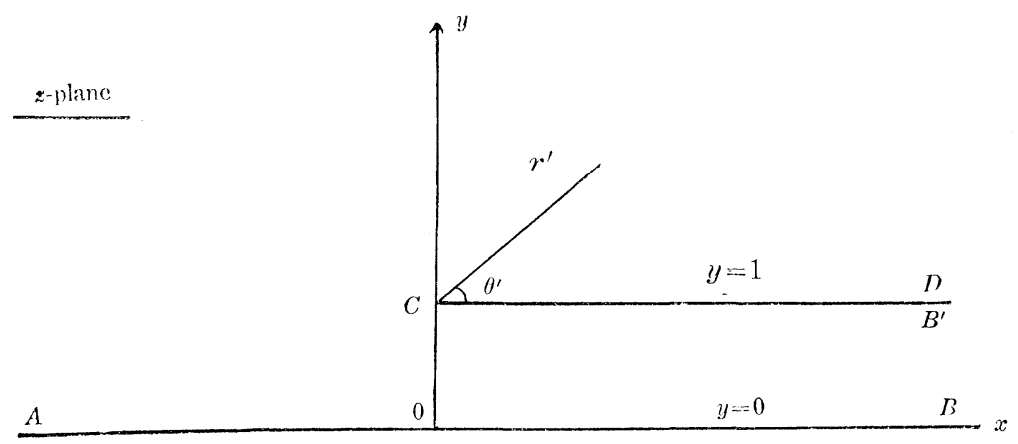

Fig. 1.

We shall here consider the slow two-dimensional flow of a viscous incompressible fluid bounded by the infinite plane $A B$, and the semiinfinite plane $C D$, as shown in Fig. 1. The motion at great distances from the planes is that of uniform shearing, and between the planes is that due to a uniform pressure gradient. W. R. Dean [1] has considered a similar type of boundary, but with the flow between the planes at great distance from the origin being due to a constant pressure, so that the type of motion produced here is fundamentally different, as is the method used to solve the problem.

We have to find a stream function satisfying the biharmonic equation

Received May 23, 1955. 
and giving zero velocities on the boundaries. We may take $A B, C D$ to be respectively the stream lines $\psi=0, \psi=1$, and we must also have $\frac{\partial \psi^{\prime}}{\partial n}=0$ on $A B, C D$ where $\frac{\partial}{\partial n}$ denotes the normal derivative.

That is to say, we seek solutions of

$$
\nabla_{1}^{4} \psi=0
$$

where

$$
\begin{aligned}
& \phi=0 \quad \text { when } \quad y=0, \\
& \partial \psi=0 \quad \text { when } \quad y=0 \text {, } \\
& \psi=1 \quad \text { when } \quad y=1, \\
& \frac{\partial \psi^{\prime}}{\partial y}=0 \quad \text { when } \quad y=1 \text {, }
\end{aligned}
$$

The transformation

$$
\pi z=w-\log w+i \pi-1
$$

where

$$
z=x+i y, \quad w=u+i v=r e^{i \theta},
$$

gives

$$
\pi y=v-\theta+\pi, \quad \pi x=u-\log r-1
$$

and so transforms the area in the upper half of the $z$-plane in Fig. 1 into the upper half of the $w$-plane as shown in Fig. 2, the boundaries becoming the real axis of the $w$-plane.

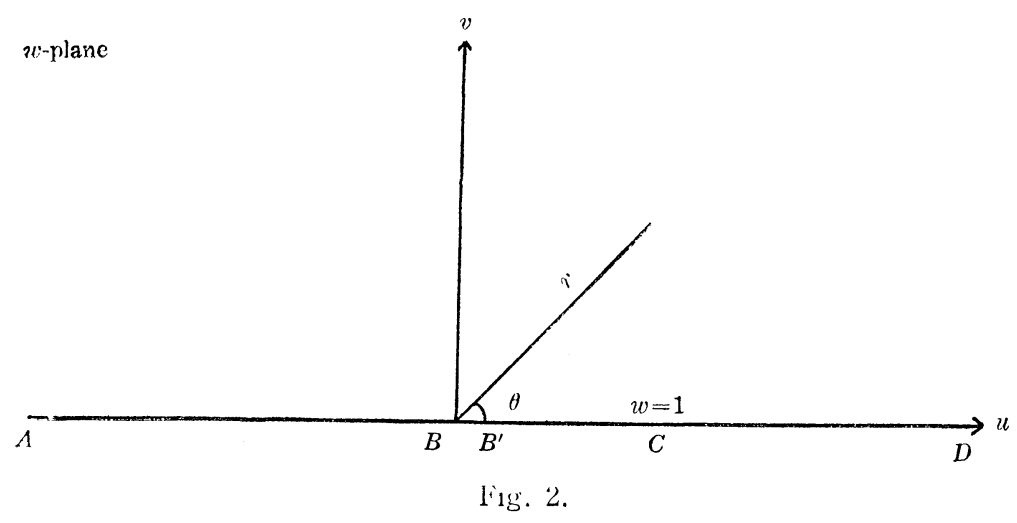


Now we have

$$
\frac{\partial}{\partial y}=\frac{\partial u}{\partial y} \frac{\partial}{\partial u}+\frac{\partial v}{\partial y} \frac{\partial}{\partial v},
$$

where

$$
\frac{\partial u}{\partial y}+i \frac{\partial v}{\partial y}=\frac{\partial w}{\partial y}=i \frac{d w}{d z}=i \pi \frac{w}{w-1}
$$

Therefore when

$$
v=0, \quad \frac{\partial u}{\partial y}=0, \quad \frac{\partial v}{\partial y}=\pi \begin{gathered}
u \\
u-1
\end{gathered}
$$

and so

$$
\left[\begin{array}{l}
\partial \psi \\
\partial y
\end{array}\right]_{v=0}=\frac{\pi u}{u-1}\left[\begin{array}{c}
\partial \psi \\
\partial v
\end{array}\right]_{v=0}
$$

A satisfactory solution is

$$
\psi=y^{2}+y(V-U)+U,
$$

provided that $U$ and $V$ are harmonic functions, chosen so that $y(V-U)+U$ does not tend to infinity as $y$ tends to infinity.

We easily see that on $A B$

$$
y=0, \quad \theta=\pi, \quad \phi=0, \quad \text { so that }[U]_{\theta=\pi}=0,
$$

and on $B^{\prime} C D$

$$
y=1, \quad \theta=0, \quad \psi=1, \quad \text { so that }[V]_{\theta=0}=0 .
$$

Also we have

$$
\frac{\partial \psi}{\partial y}=2 y+y\left(\frac{\partial V}{\partial y}-\frac{\partial U}{\partial y}\right)+\frac{\partial U}{\partial y}+V-U
$$

so that when $y=0, \theta=\pi$,

$$
\frac{\partial \psi}{\partial y}=\frac{\pi u}{u-1} \partial{ }^{2} v+V=E \quad \text { say },
$$

and when $y=1, \theta=0$,

$$
\frac{\partial \psi}{\partial y}=2+\frac{\pi u}{u-1} \frac{\partial V}{\partial v}-U=F \quad \text { say . }
$$

The boundary conditions require that equations (6) and (6.1) are satisfied, together with $E=F=0$.

It has not been found possible to find functions such that all the boundary conditions are satisfied exactly. However, expressions are 
determined for $U$ and $V$ which will satisfy (6) and (6.1) exactly, and the arbitrary constants contained in them will be chosen so that $E$ and $F$ are as small as possible at all points of the boundary. Physically this means that in the fluid motion represented by the solution there will be a small velocity of slip along the boundaries, which can be made as small as desired.

Let

$$
U=U_{1}+U_{2}, \quad V=V_{1}+V_{2},
$$

where

$$
\begin{aligned}
U_{1} & =A \mathscr{F}\left[\log \frac{2 w^{1 / 2}}{i+w^{1 / 2}}\right]^{3}+B \mathscr{Y}\left[\log \frac{2 w^{1 / 2}}{i+w^{1 / 2}}\right]^{2} \\
& +C \mathscr{S}\left[\log \frac{2 w^{1 / 2}}{i+w^{1 / 2}}\right]+D[\log w-i \pi], \\
U_{2} & =\mathscr{F} \sum_{n=1}^{\infty} a_{n}\left[\frac{1+i w^{1 / 2}}{1-i w^{1 / 2}}\right]^{n}, \\
V_{1} & =A \mathscr{Y}\left[\log \frac{2 w^{1 / 2}}{1+w^{1 / 2}}\right]^{3}+B \mathscr{S}\left[\log \frac{2 w^{1 / 2}}{1+w^{1 / 2}}\right]^{2} \\
& +G \mathscr{S}\left[\log \frac{2 w^{1 / 2}}{1+w^{1 / 2}}\right]+H \mathscr{J} \log w, \\
V_{2} & =\mathscr{F} \sum_{n=1}^{\infty} b_{n}\left[\frac{1-w^{1 / 2}}{1+w^{1 / 2}}\right]^{n} .
\end{aligned}
$$

$A, B, C, D, H, G, a_{n}, b_{n}$ are real constants to be determined.

It is assumed that the amplitude of $w^{1 / 2}$ is between 0 and $\pi / 2$ and those of $\log w^{1 / 2}, \log w, \log \left(1+w^{1 / 2}\right), \log \left(i+w^{1 / 2}\right)$ between 0 and $\pi$. These expressions satisfy (6) and (6.1) exactly at any point of the boundary, for on $A B, w^{1 / 2}=i r^{1 / 2}$ so that the functions $\log \left(\begin{array}{c}2 w^{1 / 2} \\ i+w^{1 / 2}\end{array}\right), \begin{aligned} & 1+i w^{1 / 2} \\ & 1-i w^{1 / 2}\end{aligned}$, are real, and on $B^{\prime} C D w^{1 / 2}=r^{1 / 2}$, giving real values to $\log \frac{2 w^{1 / 2}}{1+w^{1 / 2}}, \begin{aligned} & 1-w^{1 / 2} \\ & 1+w^{1 / 2}\end{aligned}$. Now we have

$$
\frac{d}{d w}\left[\log \begin{array}{c}
2 w^{1 / 2} \\
i+w^{1 / 2}
\end{array}\right]=\frac{i}{2 w\left(i+w^{1 / 2}\right)}
$$

and

$$
d w\left[\begin{array}{c}
1+i w^{1 / 2} \\
1-i w^{1 / 2}
\end{array}\right]=\frac{i}{w^{1 / 2}\left(1-i w^{1 / 2}\right)^{2}} .
$$

Using these results and the fact that 


$$
\partial U={ }^{\partial} v=\frac{d}{d w} f(w)
$$

where

$$
U=\mathscr{f} f(w),
$$

we obtain

$$
\begin{aligned}
\frac{\partial U_{1}}{\partial v}= & -\frac{3}{2} A \frac{1}{w\left(i+w^{1 / 2}\right)}\left[\log \frac{2 w^{1 / 2}}{i+w^{1 / 2}}\right]^{2} \\
& -B \circlearrowleft \frac{1}{w\left(i+w^{1 / 2}\right)} \log \left[\frac{2 w^{1 / 2}}{i+w^{1 / 2}}\right] \\
& -\frac{C}{2} \frac{1}{w\left(i+w^{1 / 2}\right)}+D v^{i}, \\
\partial U_{2} & -\sum_{n=1}^{\infty} \frac{n a_{n}}{w^{1 / 2}\left(1-i w^{1 / 2}\right)^{2}}\left[\begin{array}{c}
1+i w^{1 / 2} \\
1-i w^{1 / 2}
\end{array}\right]^{n-1},
\end{aligned}
$$

In a similar manner, we get

$$
\begin{aligned}
& { }^{\partial V_{1}}=\frac{3}{2} A \mathscr{Y}_{w\left(1+w^{1 / 2}\right)}^{i}\left[\begin{array}{cc}
\log & 2 w^{1 / 2} \\
1+w^{1 / 2}
\end{array}\right]^{2} \\
& +B \mathscr{F} \frac{i}{w\left(1+w^{1 / 2}\right)} \log \frac{2 w^{1 / 2}}{1+w^{1 / 2}} \\
& +\frac{G}{2}=\frac{i}{w\left(1+w^{1 / 2}\right)}+H=\frac{i}{w}, \\
& \frac{\partial V_{2}}{\partial v}=\mathscr{S} \sum_{n=1}^{\infty} \frac{-i n b_{n}}{w^{1 / 2}\left(1+w^{1 / 2}\right)^{2}}\left[\begin{array}{c}
1-w^{1 / 2} \\
1+w^{1 / 2}
\end{array}\right]^{n-1} \text {. }
\end{aligned}
$$

We note that on $A B$

$$
w=u=-r, \quad w^{1 / 2}=i r^{1 / 2},
$$

and

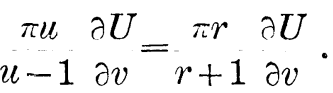

Hence we obtain

$$
\begin{aligned}
& r+1\left[\begin{array}{l}
\partial U \\
\partial v
\end{array}\right]_{\theta=\pi}=\frac{-3 A \pi}{2\left(1+r^{1 / 2}\right)(1+r)}\left[\begin{array}{lc}
\log & 2 r^{1 / 2} \\
1+r^{1 / 2}
\end{array}\right]^{2} \\
& -\underset{\left(1+r^{1 / 2}\right)(1+r)}{B \pi}\left[\log \frac{2 r^{1 / 2}}{1+r^{1 / 2}}\right]-\underset{2\left(1+r^{1 / 2}\right)(1+r)}{C \pi} \\
& -\frac{D \pi}{(1+r)}+\pi \sum_{n=1}^{\infty} \begin{array}{c}
n a_{n} r^{r^{1 / 2}} \\
(1+r)\left(1+r^{1 / 2}\right)^{2}
\end{array}\left[\frac{1-r^{r / 2}}{1+r^{1 / 2}}\right]^{n-1} \text {. }
\end{aligned}
$$


Now put

$$
r^{1 / 2}=\frac{1-p}{1+p}
$$

then as $r$ varies between 0,1 and $+\infty, p$ takes values between 1,0 and -1 . Also we see that

$$
\begin{aligned}
& 1+r^{1 / 2}=\frac{2}{(1+p)}, \quad 1+r=\frac{2\left(1+p^{2}\right)}{(1+p)^{2}}, \\
& \left(1+r^{1 / 2}\right)(1+r)=\frac{4\left(1+p^{2}\right)}{(1+p)^{3}}, \quad \begin{array}{c}
2 r^{1 / 2} \\
1+r^{1 / 2}
\end{array}=(1-p), \\
& \begin{array}{c}
r^{1 / 2} \\
(1+r)\left(1+r^{1 / 2}\right)
\end{array}=\begin{array}{c}
(1-p)(1+p)^{2} \\
4\left(1+p^{2}\right)
\end{array}, \quad \begin{array}{c}
r^{1 / 2} \\
(1+r)\left(1+r^{1 / 2}\right)^{2}
\end{array}=\begin{array}{c}
(1-p)(1+p)^{3} \\
8\left(1+p^{2}\right)
\end{array},
\end{aligned}
$$

leading to

$$
\begin{aligned}
\frac{\pi r}{r+1}\left[\frac{\partial U}{\partial v}\right]_{\ni=\pi}=-\frac{3}{8} A \pi \frac{(1+p)^{3}}{\left(1+p^{2}\right)}[\log (1-p)]^{2} \\
-B \frac{\pi}{4} \frac{(1+p)^{3}}{\left(1+p^{2}\right)} \log (1-p)-C^{\pi} \frac{(1+p)^{3}}{8} \\
-D \frac{\pi\left(1+p^{2}\right)}{2} \frac{(1+p)^{2}}{\left(1+p^{2}\right)}+\pi \sum_{n=1}^{\infty} \frac{n}{8} a_{n} p^{n-1} \frac{(1-p)(1+p)^{3}}{\left(1+p^{2}\right)} .
\end{aligned}
$$

For $V_{1}$ we have

$$
\begin{aligned}
{\left[V_{1}\right]_{9=\pi} } & =A \circlearrowleft\left[\log \frac{2 i r^{1 / 2}}{1+i r^{1 / 2}}\right]^{3}+B \circlearrowleft\left[\log \frac{2 i r^{1 / 2}}{1+i r^{1 / 2}}\right]^{2} \\
& +G \circlearrowleft\left[\log \frac{2 i r^{1 / 2}}{1+i r^{1 / 2}}\right]+H \pi,
\end{aligned}
$$

where

$$
\log \left[\frac{2 i r^{1 / 2}}{1+i r^{1 / 2}}\right]=\log 2+i \frac{\pi}{2}+\log (1-p)-\log [(1+p)+i(1-p)] .
$$

It is easy to see that

$$
\begin{aligned}
\log [1+p+i(1-p)] & =\frac{1}{2} \log \left[2\left(1+p^{2}\right)\right]+i \tan ^{-1} \frac{1-p}{1+p} \\
& =\frac{1}{2} \log 2+\frac{1}{2} \log \left(1+p^{2}\right)+i\left(\frac{\pi}{4}-\tan ^{-1} p\right),
\end{aligned}
$$

and so

$$
\begin{aligned}
\log \frac{2 i r^{1 / 2}}{1+i r^{1 / 2}} & =\frac{1}{2} \log 2+\log (1-p)-\frac{1}{2} \log \left(1+p^{2}\right)+i\left(\frac{\pi}{4}+\tan ^{-1} p\right) \\
& =\alpha+i \beta, \quad \text { say . }
\end{aligned}
$$


We can thus write

$$
\left[V_{1}\right]_{\theta=\pi}=A\left(3 \alpha^{2} \beta-\beta^{3}\right)+2 B \alpha \beta+G \beta+H \pi
$$

In the same way we get

$$
\begin{aligned}
{\left[V_{2}\right]_{\theta=\pi} } & =\mathscr{F} \sum_{n=1}^{\infty} b_{n}\left[\frac{1-i r^{1 / 2}}{1+i r^{1 / 2}}\right]^{n} \\
& =\mathscr{J} \sum_{n=1}^{\infty} b_{n}\left[\begin{array}{c}
1+p-i(1-p) \\
1+p+i(1-p)
\end{array}\right]^{n} \\
& =\mathscr{F} \sum_{n=1}^{\infty} b_{n}\left[\frac{2 p-i\left(1-p^{2}\right)}{1+p^{2}}\right]^{n}
\end{aligned}
$$

Subsituting these values in the expression for $E$, the velocity of slip along $A B$ is

$$
\begin{aligned}
E= & -\frac{3}{8} A \pi \frac{(1+p)^{3}}{1+p^{2}}[\log (1-p)]^{2}-B \frac{\pi(1+p)^{3}}{4} \log (1-p) \\
& -C \frac{\pi(1+p)^{3}}{81+p^{2}}-D \frac{\pi(1+p)^{2}}{2} \frac{\left(1+p^{2}\right.}{1+\pi \sum_{n=1}^{\infty} \frac{n}{8} a_{n} p^{n-1}(1-p)(1+p)^{3}}\left(1+p^{2}\right) \\
& +A\left(3 \alpha^{2} \beta-\beta^{3}\right)+2 B \alpha \beta+G \beta+H \pi+. \sum_{n=1}^{\infty} b_{n}\left[\begin{array}{c}
2 p-i\left(1-p^{2}\right) \\
1+p^{2}
\end{array}\right]^{n} .
\end{aligned}
$$

We shall chose the constants involved so that $E=0$ when $p= \pm 1$, and then expand $E$ in powers of $p$. Equating to zero the confficients of $p^{0}$, $p^{1}, p^{2}$, etc. we will thus obtain a set of simultaneous equations for the determination of the arbitrary constants. By this procedure $E$ can be made as small as we please in the range $-1 \leqq p \leqq 1$. When $p=-1$, we see that

$$
\beta=0
$$

and

$$
E=H \pi
$$

so therefore

$$
H=0 \text {. }
$$

As $p$ tends to 1 , we see that $\beta$ tends to $\frac{\pi}{2}$, and $\alpha$ can be taken to be

$$
\log _{e}(1-p)=l, \quad \text { say, }
$$

so that $E$ approximates to

$$
-3 A \frac{\pi}{2} l^{2}-B \pi l-C \frac{\pi}{2}-D \pi+A\left(3 \frac{\pi}{2} l^{2}-\frac{\pi^{3}}{8}\right)+B \pi l+G \frac{\pi}{2} .
$$


'Therefore we must have

$$
A \pi_{8}^{\pi^{3}}+D \pi+C_{2}^{\pi}-G \frac{\pi}{2}=0 .
$$

Before continuing with the calculation by expanding in terms of $p$, it has been found convenient at this stage to obtain a similar type of expression for $F$. Along the boundary $B^{\prime} C D$ we have $w=u=r$, so that

$$
w^{1 / 2}=r^{1 / 2} \text {. }
$$

Now set

$$
r^{1 / 2}=\begin{aligned}
& 1-s \\
& 1+s
\end{aligned}
$$

so that as $r^{1 / 2}$ varies between 0,1 , and $+\infty, s$ takes values between 1,0 , and -1 . Using the relations

$$
\begin{gathered}
r-1=-\frac{4 s}{(1+s)^{2}}, \quad(r-1)\left(1+r^{1 / 2}\right)=-\frac{8 s}{(1+s)^{3}}, \\
(r-1)\left(1+r^{1 / 2}\right)
\end{gathered}=-\begin{array}{cc}
(1-s)(1+s)^{2} \\
8 s
\end{array}, \quad\left(1+r^{1 / 2}\right)^{2 / 2}(r-1)=-\left(\begin{array}{c}
1-s)(1+s)^{3}, \\
16 s
\end{array}\right.
$$

we get

$$
\begin{aligned}
\frac{\pi u}{(u-1)} & {\left[\frac{\partial V}{\partial v}\right]_{\theta=0}=-{ }_{16 s}^{3 A \pi}(1+s)^{3}[\log (1-s)]^{2} } \\
& -\frac{B \pi}{8 s}(1+s)^{3} \log (1-s)-{ }_{16 s}^{G \pi}(1+s)^{3} \\
& -\frac{H \pi}{4 s}(1+s)^{2}+\sum_{n=1}^{\infty} \frac{n \pi}{16} b_{n}(1-s)(1+s)^{3} s^{n-2} .
\end{aligned}
$$

Again, we have

$$
\begin{aligned}
\log \frac{2 r^{1 / 2}}{i+r^{1 / 2}} & =\log 2+\log (1-s)-\log [(1-s)+i(1+s)], \\
& =\log 2+\log (1-s)-\frac{1}{2} \log \left(1+s^{2}\right)-i\left(\frac{\pi}{4}+\tan ^{-1} s\right), \\
& =\alpha-i \beta .
\end{aligned}
$$

so that

$$
[U]_{\theta=0}=A\left(\beta^{3}-3 \alpha^{2} \beta\right)-2 B \alpha \beta-C \beta-\pi D+\mathscr{J} \sum_{n=1}^{\infty} a_{n}\left[\begin{array}{c}
1+s+i(1-s) \\
1+s-i(1-s)
\end{array}\right]^{n} .
$$

Finally we obtain 
$(13.2)$

$$
\begin{aligned}
F^{\prime}=2- & 3 A \pi(1+s)[\log (1-s)]^{2}-\frac{B \pi}{8 s}(1+s)^{3} \log (1-s) \\
& -\frac{G \pi}{16 s}(1+s)^{3}-H \pi(1+s)^{2}+\sum_{n=1}^{\infty} \frac{n \pi}{16} b_{n}(1-s)(1+s)^{3} s^{n-2} \\
& +A\left(3 \alpha^{2} \beta-\beta^{3}\right)+2 B \alpha \beta+C \beta+\pi D-Y \sum_{n=1}^{\infty} a_{n}\left[\begin{array}{c}
2 s+i\left(1-s^{2}\right) \\
1+s^{2}
\end{array}\right]^{n} .
\end{aligned}
$$

We must have $F=0$ when $s= \pm 1$, and also $F^{\prime}$ must be finite when $s=0$. When $s=-1$,

$$
F=2+\pi D,
$$

and since this must vanish,

$$
D=-2 / \pi
$$

As $s$ tends to $1, \beta$ tends to $\pi / 2$, and $\alpha$ may be taken to be $\log _{e}(1-s)=l$ as before, so that $F$ approximates to

$$
2-{ }_{2}^{3} \pi A l^{2}-B \pi l-{ }_{2}^{\pi} G-H \pi+A\left[33_{2}^{\pi} l^{3}-\frac{\pi^{3}}{8}\right]+B \pi l+C_{2}^{\pi}+\pi D .
$$

Using equations (11) and (14), we see that

$$
G_{2}^{\pi}-C_{2}^{\pi}+A_{8}^{\pi^{3}}=0
$$

Also we notice that $F$ contains the term $\frac{\pi}{16 s}\left(b_{1}-G\right)$, and since $F$ is to be finite when $s=0$, we must have

$$
b_{1}=G \text {. }
$$

Adding equations (12) and (15) we get

$$
A_{4}^{\pi^{3}}+D \pi=0
$$

giving

$$
A=\frac{8}{\pi^{3}} .
$$

We also have from (15)

$$
C=b_{1}+\frac{2}{\pi} \text {. }
$$

Using these results, the velocities of slip on $A B$ and $B^{\prime} C D$ become 
(19)

$$
\begin{aligned}
E= & -\frac{3(1+p)^{3}}{\pi^{2}\left(1+p^{2}\right)}[\log (1-p)]^{2}-B \frac{\pi(1+p)^{3}}{4\left(1+p^{2}\right)} \log (1-p) \\
& +\frac{1(1+p)^{2}(3-p)}{4}\left(1+b_{1}\left[\beta-\frac{\pi(1+p)^{3}}{8\left(1+p^{2}\right)}\right]+\frac{8}{\pi^{3}}\left(3 \alpha^{2} \beta-\beta^{3}\right)+2 B \alpha \beta\right. \\
& +\mathscr{F} \sum_{n=1}^{\infty} b_{n}\left[\frac{2 p-i\left(1-p^{2}\right)}{1+p^{2}}\right]+\sum_{n=1}^{\infty} a_{n} \frac{\pi}{8} p^{n-1}(1-p) \frac{(1+p)^{3}}{1+p^{2}}, \\
F= & -\frac{3}{2 \pi^{2}} s{ }^{2}(1+s)^{3}[\log (1-s)]^{2}-B \frac{\pi}{8}(1+s)^{3} \log (1-s) \\
& +b_{1}\left[\beta-\frac{\pi}{16}(1+s)^{3}\right]+2 \frac{\beta}{\pi}+\frac{8}{\pi^{3}}\left(3 \alpha^{2} \beta-\beta^{3}\right)+2 B \alpha \beta \\
& +\sum_{n=2}^{\infty} n \pi b_{n}(1-s)(1+s)^{3} s^{n-2}-\sum_{n=1}^{\infty} a_{n}\left[\frac{\left.2 s+i\left(1-s^{2}\right)\right]^{n} .}{1+s^{2}}\right]
\end{aligned}
$$

As a first approximation, we will restrict the infinite series to four terms each. We will expand these results for $E$ and $F$ in powers of $p$ and $s$, equate to zero the coefficients of $p^{3}, p^{1}, p^{2}$, and $s^{0}, s^{1}, s^{2}$, and ignoring the remaining terms of $O\left(p^{3}\right), O\left(s^{3}\right)$, we will get a set of simultaneous equations for which $a_{1}, a_{2}, a_{3}, b_{1}, b_{2}, b_{3}$, are determined in terms of $a_{t}, b_{1}$ and $B$. The conditions at infinity will give the values of $a_{1}, b_{t}$, in terms of $B$. If greater accuracy is required more terms may be included in the series and higher powers of $p$ and $s$ neglected. Expanding $E$ in powers of $p$ as far as $p^{2}$, we have

$$
\begin{aligned}
E= & -\frac{3}{\pi^{2}}\left(p^{2}\right)+B \frac{\pi}{4}\left(p+\frac{7}{2} p^{2}\right)+\frac{1}{4}\left(3+5 p-2 p^{2}\right) \\
& +b_{1}\left[\frac{\pi}{4}+p-\frac{\pi}{8}\left(1+3 p+2 p^{2}\right)\right]+\frac{8}{\pi^{3}}\left\{3 \left[t^{2} \frac{\pi}{4}+p\left(t^{2}-\frac{\pi}{2} t\right)\right.\right. \\
& \left.\left.+p^{2}\left(\frac{\pi}{4}-\frac{\pi}{2} t-2 t\right)\right]-\frac{\pi^{3}}{64}-3 \frac{\pi^{2}}{16} p-3 \frac{\pi}{4} p^{2}\right\}+2 B\left[t \frac{\pi}{4}+p\left(t-\frac{\pi}{4}\right)\right. \\
& \left.-p^{2}\left(\frac{\pi}{4}+1\right)\right]+a_{1} \frac{\pi}{8}\left(1+2 p-p^{2}\right)+a_{2} \frac{\pi}{4}\left(p+2 p^{2}\right)+3 \frac{\pi}{8}_{3}^{\pi} a_{3} p^{2} \\
& -b_{1}\left(1-2 p^{2}\right)-b_{2} 4 p+b_{3}\left(1-18 p^{2}\right)+b_{4} 8 p,
\end{aligned}
$$

where

$$
t=\frac{1}{2} \log _{e} 2
$$

Equating to zero the coefficients of $p^{0}, p^{1}, p^{2}$, we have the equations 
(21)

$$
\begin{aligned}
b_{1}\left(\frac{\pi}{8}-1\right)+\frac{\pi}{8} a_{1}+b_{3} & =-\frac{5}{8}-6 \frac{t^{2}}{\pi^{2}}-t \frac{\pi}{2} B, \\
& =-0.69802-0.54441 B,
\end{aligned}
$$

(22)

$$
\begin{aligned}
b_{1}\left(1-3 \frac{\pi}{8}\right)+\frac{\pi}{4} a_{1}+\frac{\pi}{4} a_{2}-4 b_{2}= & -\frac{5}{4}-\frac{3}{\pi}\left(8 \frac{t^{2}}{\pi^{2}}-4 \frac{t}{\pi}-\frac{1}{2}\right) \\
& +B\left(\frac{\pi}{4}-2 t\right)-8 b_{4}, \\
= & -0.44416+0.09224 B-8 b_{1},
\end{aligned}
$$

(23)

$$
\begin{aligned}
b_{1}\left(2-\frac{\pi}{4}\right)-18 b_{3}-\frac{\pi}{8} a_{1}+\frac{\pi}{2} a_{2}+ & 3 \frac{\pi}{8} a_{3}=\frac{3}{\pi^{2}}+\frac{1}{2}-\frac{3}{\pi}\left(\frac{2}{\pi}-4 \frac{t}{\pi}-16{ }_{\pi^{2}}^{t}\right) \\
& +\frac{6}{\pi^{2}}+B\left(2+\frac{\pi}{2}-\frac{7}{8} \pi\right), \\
= & 1.76168+0.82193 B .
\end{aligned}
$$

Similarly expanding $F$ in powers of $s$, as far as $s^{2}$, we get

$$
\begin{aligned}
F= & -\frac{3}{2 \pi^{2}}\left(s+4 s^{2}\right)+B \frac{\pi}{8}\left(1+\frac{7}{2} s+\frac{29}{6} s^{2}\right)+\frac{1}{2}+\frac{2 s}{\pi} \\
& +b_{1}\left[\frac{\pi}{4}+s-\frac{\pi}{16}\left(1+3 s+3 s^{2}\right)\right]+b_{2} \frac{\pi}{8}(1+2 s)+b_{3} \frac{3 \pi}{16}\left(s+2 s^{2}\right) \\
& +b_{4} \frac{\pi}{4} s^{2}+\frac{8}{\pi^{3}}\left\{3\left[t^{2} \frac{\pi}{4}+s\left(t^{2}-\frac{\pi}{2} t\right)+s^{2}\left(\frac{\pi}{4}-\frac{\pi}{2} t-2 t\right)\right]\right. \\
& \left.-\frac{\pi^{3}}{64}-3 \frac{\pi^{2}}{16} s-3 \frac{\pi}{4} s^{2}\right\}+2 B\left[t \frac{\pi}{4}+s\left(t-\frac{\pi}{4}\right)-s^{2}\left(\frac{\pi}{4}+1\right)\right] \\
& -a_{1}\left(1-2 s^{2}\right)-4 a_{2} s-a_{3}\left(-1+18 s^{2}\right)+8 a_{1} s .
\end{aligned}
$$

Equating to zero the coefficient of $s^{0}, s^{1}, s^{2}$, we get

$$
\begin{aligned}
33_{16}^{\pi} b_{1}+\frac{\pi}{8} b_{2}-a_{1}+a_{3}= & -\frac{1}{2}-6 \frac{t^{2}}{\pi^{2}}+\frac{1}{8}-B\left(\frac{\pi}{8}+t \frac{\pi}{2}\right) \\
= & -0.44802-0.93711 B . \\
b_{1}\left(1-3 \frac{\pi}{16}\right)+{ }_{4}^{\pi} b_{2}+3 \frac{\pi}{16} b_{3}-4 a_{2}= & 3\left(\frac{1}{\pi}-8 \frac{t^{2}}{\pi^{2}}+4 \frac{t}{\pi}\right) \\
& -\frac{1}{2 \pi}-B\left(2 t-\frac{\pi}{16}\right)-8 a_{1}, \\
= & 0.32121-0.49681 B-8 a_{4} .
\end{aligned}
$$


(26)

$$
\begin{aligned}
-3 \frac{\pi}{16} b_{1}+3 \frac{\pi}{8} b_{3}+2 a_{1}-18 a_{3}= & \frac{3}{\pi}\left(\frac{2}{\pi}+1 \frac{t}{\pi}+16 \frac{{ }^{2}}{t}\right) \\
& +B\left(2-\frac{5}{48} \pi\right)-{ }_{4}^{\pi} b_{4}, \\
= & 1.56564+1.67275 B-\frac{\pi}{4} b_{4} .
\end{aligned}
$$

Solving the equations (21)-(26) we obtain

$$
\begin{aligned}
& a_{1}=2.41918+3.29530 B+0.88575 a_{1}+2.18575 b_{4}, \\
& a_{2}=0.32523+0.60601 B+2.24986 a_{4}+0.67766 b_{4}, \\
& a_{3}=0.09389+0.17611 B+0.08255 a_{4}+0.24184 b_{4}, \\
& b_{1}=2.83764+3.30740 B+0.98227 a_{4}+1.64053 b_{1}, \\
& b_{2}=0.52365+0.59576 B+0.57194 a_{4}+2.48922 b_{4}, \\
& b_{3}=0.07535+0.17003 B+0.24870 a_{4}+0.13799 b_{4}
\end{aligned}
$$

The coefficients $a_{t}$ and $b_{t}$ will be found in terms of $B$ by considering the conditions for $\psi$ when $|w|$ is large. $B$ will be chosen so that $E$ and $F$ are small at all points of the boundary.

When $w$ is large, we have the following expansions:-

$$
\begin{aligned}
& \log \begin{array}{c}
2 w^{1 / 2} \\
1+w^{1 / 2}
\end{array}=\log 2-\left[w^{-1 / 2}-1 w^{-1}+O\left(w^{-3 / 2}\right)\right], \\
& \log \frac{2 w^{1 / 2}}{i+w^{1 / 2}}=\log 2-\left[i w^{-1 / 2}+\frac{1}{2} w^{-1}+O\left(w^{-3 / 2}\right)\right], \\
& {\left[\log \frac{2 w^{1 / 2}}{1+w^{1 / 2}}\right]^{2}=(\log 2)^{2}-2 \log 2 w^{-1 / 2}+(1+\log 2) w^{-1}+O\left(w^{-3 / 2}\right),} \\
& {\left[\log \frac{2 w^{1 / 2}}{i+w^{1 / 2}}\right]^{2}=(\log 2)^{2}-2 i \log 2 w^{-1 / 2}-(1+\log 2) w^{-1}+O\left(w^{-3 / 2}\right),} \\
& {\left[\log \frac{2 w^{1 / 2}}{1+w^{1 / 2}}\right]^{3}=(\log 2)^{3}-3(\log 2)^{2} w^{-1 / 2}+\left[\begin{array}{l}
3 \\
2
\end{array}(\log 2)^{2}+3 \log 2\right] w^{-1}} \\
& {\left[\log \frac{2 w^{1 / 2}}{i+w^{1 / 2}}\right]^{3}=(\log 2)^{3}-3 i(\log 2)^{2} w^{-1 / 2}-\left[\begin{array}{l}
3 \\
2
\end{array}(\log 2)^{2}+3 \log 2\right] w^{-1}} \\
& \quad+O\left(w^{-3 / 2}\right) .
\end{aligned}
$$

On setting $L=\log 2$, and neglecting terms $O\left(w^{-3 / 2}\right)$, we get 


$$
\begin{aligned}
V_{1}-U_{1}= & \mathscr{F}\left\{\begin{array}{l}
8 \\
\pi^{2}
\end{array}\left[3(i-1) L^{2} w^{-1 / 2}+3\left(L^{2}+2 L\right) w^{-1}\right]\right. \\
& +B\left[2(i-1) L w^{-1 / 2}+2(1+L) w^{-1}\right]+b_{1}\left[(i-1) w^{-1 / 2}+w^{-1}\right] \\
& \left.+\frac{2}{\pi}\left[i w^{-1 / 2}+\frac{1}{2} w^{-1}\right]\right\}+\frac{2}{\pi}(\theta-\pi), \\
= & \mathscr{F}\left\{w^{1 / 2}(i-1)\left[24 \frac{L^{2}}{\pi^{3}}+2 L B+b_{1}\right]+{ }_{\pi}^{2 i} w^{-1 / 2}\right. \\
& \left.+w^{-1}\left[\begin{array}{c}
24 \\
\pi^{3}
\end{array}\left(L^{2}+2 L\right)+2(1+L) B+b_{1}+\frac{1}{\pi}\right]\right\}+{ }_{\pi}^{2}(\theta-\pi) .
\end{aligned}
$$

Again we have

$$
\begin{aligned}
{\left[\begin{array}{l}
1-w^{1 / 2} \\
1+w^{1 / 2}
\end{array}\right]^{n} } & =(-1)^{n}\left[\frac{1-w^{-1 / 2}}{1+w^{-1 / 2}}\right]^{n}, \\
& =(-1)^{n}\left[1-2 n w^{-1 / 2}+2 n^{2} w^{-1}+O\left(w^{-3 / 2}\right)\right],
\end{aligned}
$$

and

$$
\left[\begin{array}{c}
1+i w^{1 / 2} \\
1-i w^{1 / 2}
\end{array}\right]^{n}=(-1)^{n}\left[1-2 n i w^{-1 / 2}-2 n^{2} w^{-1}+O\left(w^{-3 / 2}\right)\right]
$$

so that

$$
\begin{aligned}
V_{2}-U_{2}=\mathscr{F}\{ & \left(2 b_{1}-4 b_{2}+6 b_{3}-8 b_{4}\right) w^{-1 / 2}-\left(2 a_{1}-4 a_{2}+6 a_{3}-8 a_{4}\right) i w^{-1 / 2} \\
& \left.+w^{-1}\left(2 b_{1}-8 b_{2}+18 b_{3}-32 b_{4}+2 a_{1}-8 a_{2}+18 a_{3}-32 a_{4}\right)\right\},
\end{aligned}
$$

neglecting $O\left(w^{-3 / 2}\right)$. Also when $y$ is large

$$
U \rightarrow-\frac{2}{\pi}(\theta-\pi) \text {. }
$$

Now we know that $\psi$ must tend to $y^{2}$ when $y$ is large. This means that the coefficients of $y w^{-1 / 2}$ and $i y w^{-1 / 2}$ in $y\left(V_{1}-U_{1}+V_{2}-U_{2}\right)$ must vanish. We therefore have

$$
24 \frac{L^{2}}{\pi^{3}}+2 L B+b_{1}+\frac{2}{\pi}-\left(2 a_{1}-4 a_{2}+6 a_{3}-8 a_{4}\right)=0,
$$

and

$$
24 \frac{L^{2}}{\pi^{3}}+2 L B+b_{1}-\left(2 b_{1}-4 b_{2}+6 b_{3}-8 b_{4}\right)=0 .
$$

Using equations (27) these last two equations become

$$
0.25461+0.52952 B-15.71491 a_{4}+1.47137 b_{4}=0,
$$

$$
0.82323+0.55824 B+0.18671 a_{4}-15.48841 b_{1}=0,
$$


which give

$$
a_{4}=0.02120+0.03712 B \text {, }
$$

and

$$
b_{4}=0.05341+0.03641 B \text {. }
$$

Also $y \mathscr{F} w^{-1}$ is proportional to $\sin ^{2} \theta$ when $y$ is large, so that we see that the stream function tends to

$$
\begin{aligned}
\psi=y^{2} & +y\left(2 \frac{\theta}{\pi}-2\right)+(\text { Constant }) \sin ^{2} \theta+2-2 \frac{\theta}{\pi}, \\
= & y\left(2 \frac{v}{\pi}-y\right)+(\text { Constant }) \sin ^{2} \theta+2-2 \frac{\theta}{\pi} .
\end{aligned}
$$

Now when $y$ is large $\frac{v}{\pi} \rightarrow y$, so that the most important term in the stream function is $y^{2}$, the other terms being always finite, thus giving uniform shearing motion above the points $A$ and $\mathrm{D}$.

With the values of $a_{4}$ and $b_{t}$ given by (30) (30.1), the other coefficients are

$$
\begin{aligned}
& a_{1}=2.55469+3.40794 B, \\
& a_{2}=0.40912+0.71426 B, \\
& a_{3}=0.10855+0.18799 B, \\
& b_{1}=2.94607+3.40372 B, \\
& b_{2}=0.66870+0.70782 B, \\
& b_{3}=0.08799+0.18430 B .
\end{aligned}
$$

With these values of $a_{n}, b_{n}, n=1,2,3,4$ the velocities of slip at different points on the boundary are calculated in terms of $B$. Table 1 gives the values of $E$ and $F$ at the points

$$
p, s: \quad \pm 0.1, \quad \pm 0.3, \quad \pm 0.7, \quad \pm 0.8, \quad \pm 0.9 .
$$

Using the method of least squares, the mean value of $B$ is -0.52 , and the velocities of slip are shown in Table 2 .

The values of $E$ and $F$ are small, and can be made still smaller if more than four terms are included in the series for $U_{2}$ and $V_{2}$.

It will be noticed that $F$ is exactly zero at the sharp edge $C$ where $s=0$. The values of the coefficients are given in Table 3 . 
Table 1.

\begin{tabular}{|c|c|c|c|}
\hline$p$ & $E$ & $s$ & $F$ \\
\hline 0.9 & $.13946+.32042 B$ & 0.9 & $0.22003+0.32776 B$ \\
0.8 & $.22416+.56262 B$ & 0.8 & $0.32998+0.50692 B$ \\
0.7 & $.18632+.53530 B$ & 0.7 & $0.33664+0.55284 B$ \\
0.3 & $.00738+.03768 B$ & 0.3 & $0.05672+0.08351 B$ \\
0.1 & $.00078+.00196 B$ & 0.1 & $0.00148+0.00192 B$ \\
-0.1 & $.00313+.00214 B$ & -0.1 & $-0.00065-0.00122 B$ \\
-0.3 & $.00128+.00930 B$ & -0.3 & $-0.00503-0.00958 B$ \\
-0.7 & $.00303+.00444 B$ & -0.7 & $-0.00063-0.00100 B$ \\
-0.8 & $.00711+.00286 B$ & -0.8 & $-0.00088-0.00152 B$ \\
-0.9 & $.00487+.00014 B$ & -0.9 & $-0.00013-0.00105 B$ \\
\hline
\end{tabular}

Table 2.

\begin{tabular}{|c|c|c|c|}
\hline$p$ & $E$ & $s$ & \multicolumn{1}{|c|}{$F$} \\
\hline 0.9 & -0.0272 & 0.9 & -0.0504 \\
0.8 & -0.0684 & 0.8 & 0.0664 \\
0.7 & -0.0920 & 0.7 & 0.0492 \\
0.3 & -0.0122 & 0.3 & 0.0133 \\
0.1 & -0.0002 & 0.1 & 0.0002 \\
-0.1 & 0.0020 & -0.1 & -0.0001 \\
-0.3 & 0.0036 & -0.3 & -0.0001 \\
-0.7 & 0.0007 & -0.7 & -0.0001 \\
-0.8 & 0.0056 & -0.8 & -0.0001 \\
-0.9 & 0.0048 & -0.9 & 0.0004 \\
\hline
\end{tabular}

Table 3.

\begin{tabular}{|c|c|r|}
\hline$n$ & $a_{n}$ & \multicolumn{1}{c|}{$b_{n}$} \\
\hline 1 & 0.78257 & 1.14148 \\
2 & 0.03771 & 0.30063 \\
3 & 0.01080 & -0.00788 \\
4 & 0.00190 & 0.03462 \\
\hline
\end{tabular}

The motion between the barriers at a great distance from 0 is considered next, that is to say the motion in the region $w=0$.

When $|w|$ is small, we have the following expansions

$$
\begin{aligned}
& \log \frac{2 w^{1 / 2}}{1+w^{1 / 2}}=\frac{1}{2} \log w+\log 2+O\left(w^{1 / 2}\right), \\
& \log \underset{i+w^{1 / 2}}{2 w^{1 / 2}}=\frac{1}{2} \log w+\log 2-\frac{1}{2} i \pi+O\left(w^{1 / 2}\right) .
\end{aligned}
$$

Also when $|w|$ is small, the transformation (4) may be taken as

$$
\pi z=-\log w+i \pi-1
$$

which gives

$$
\begin{aligned}
\log \frac{2 w^{1 / 2}}{1+w^{1 / 2}} & =\log 2+\frac{1}{2}(i \pi-1-\pi z)+O\left(w^{1 / 2}\right) \\
& =\log 2-\frac{1}{2}(1+\pi x)+\frac{i \pi}{2}(1-y)+O\left(w^{1 / 2}\right),
\end{aligned}
$$




$$
\log \frac{2 w^{1 / 2}}{i+w^{1 / 2}}=\log 2+\frac{1}{2}(1+\pi x)-\frac{i \pi}{2} y+O\left(w^{1 / 2}\right) .
$$

We thus have approximately

$$
\begin{gathered}
\mathrm{V}_{1}=A\left\{-\frac{\pi^{3}}{8}(1-y)^{3}+\frac{3 \pi}{2}\left(\log 2-\frac{1}{2}-\frac{\pi x}{2}\right)^{2}(1-y)\right\} \\
+B \pi(1-y)\left(\log 2-\frac{1}{2}-\frac{\pi x}{2}\right)+\frac{\pi G}{2}(1-y), \\
U_{1}=A\left\{\frac{\pi^{3}}{8} y^{3}-\frac{3 \pi}{2}\left(\log 2-\frac{1}{2}-\frac{\pi x}{2}\right)^{2} y\right\}-B \pi y\left(\log 2-1-\frac{\pi x}{2}-\frac{\pi}{2}\right) \\
-C \pi y-D \pi y,
\end{gathered}
$$

and

$$
\begin{aligned}
V_{1}-U_{1}= & A\left\{-\frac{\pi^{3}}{8}\left(1-3 y+3 y^{2}\right)+\frac{3 \pi}{2}\left(\log 2-\frac{1}{2}-\frac{\pi x}{2}\right)^{2}\right\} \\
& +B \pi\left[\log 2-\frac{1}{2}-\begin{array}{c}
\pi x \\
2
\end{array}\right]+y\left(\begin{array}{c}
C \pi \\
2
\end{array}+D \pi-\frac{C \pi}{2}\right)+\frac{G \pi}{2},
\end{aligned}
$$

since all other terms tend to zero as $w \rightarrow 0$ because $\underset{w \rightarrow 0}{L t} w^{1 / 2} \log w=0$. The stream function thus becomes

$$
\psi=y^{2}+A_{8}^{\pi^{3}}\left[-y+3 y^{2}-2 y^{3}\right]+y^{2}\left[C-\frac{\pi}{2}+D \pi-\frac{G \pi}{2}\right]+y\left[\begin{array}{c}
G \pi \\
2
\end{array}-C \frac{\pi}{2}-D \pi\right] .
$$

Now

$$
\begin{gathered}
A \pi^{3} \\
8
\end{gathered}=1 \text { and } \quad C \pi+D \pi-\frac{G \pi}{2}=1
$$

and so we have

$$
\psi=3 y^{2}-2 y^{3}, \quad \frac{\partial \psi}{\partial y}=6 y(1-y),
$$

This is the stream function for the flow in an infinite channel under a uniform pressure gradient with

$$
\begin{aligned}
& \psi=0 \text { when } y=0, \\
& \psi=1 \text { when } y=1,
\end{aligned}
$$

and $\begin{gathered}\partial \psi^{\prime} \\ \partial y\end{gathered}$ vanishing when $y=0, \quad y=1$.

We will now consider the motion near the sharp edge $C$. In this region $z$ is nearly $i$ and $w$ is nearly 1 , so that if we put 


$$
z=i+z^{\prime}, \quad y=1+y^{\prime}, \quad w^{1 / 2}=1+\zeta,
$$

then $z^{\prime}, y^{\prime}$ and $\zeta$ are small.

The stream function must be found to order $\zeta^{3}$. To this order the transformation gives

$$
\begin{aligned}
\pi\left(z^{\prime}+i\right) & =(1+\zeta)^{2}-2 \log (1+\zeta)+i \pi-1, \\
\pi z^{\prime} & =2 \zeta^{2}-\frac{2}{3} \zeta^{3},
\end{aligned}
$$

and so $y^{\prime}$ is of the order $\zeta^{2}$.

Now

$$
\begin{aligned}
\psi & =\left(1+y^{\prime}\right)^{2}+\left(1+y^{\prime}\right)(V-U)+U, \\
& =\left(1+y^{\prime}\right)^{2}+y^{\prime}(V-U)+V,
\end{aligned}
$$

so that we have to find $V$ to order $\zeta^{3}$ and $V-U$ to order $\zeta$.

We now use the expansions

$$
\begin{aligned}
\log w & =\log (1+\zeta)^{2} \\
& =2\left(\zeta-\frac{\zeta^{2}}{2}+\frac{\zeta^{3}}{3}+\cdots\right) \\
\log \frac{2 w^{1 / 2}}{1+w^{1 / 2}} & =\log (1+\zeta)-\log \left(1+\frac{\zeta}{2}\right) \\
& =\frac{\zeta}{2}-\frac{3}{8} \zeta^{2}+\frac{7}{24} \zeta^{3}+\cdots, \\
\log \begin{array}{c}
2 w^{1 / 2} \\
i+w^{1 / 2}
\end{array} & \frac{1}{2} \log 2-i \frac{\pi}{4}+\log (1+\zeta)-\log \left(1+\frac{1-i}{2}-\zeta\right) \\
& =\frac{1}{2} \log 2-i \frac{\pi}{4}+\frac{\zeta}{2}(1+i)+\cdots
\end{aligned}
$$

To order $\zeta$ on putting $\mathrm{t}=\frac{1}{2} \log _{e} 2$, we obtain

$$
\begin{aligned}
V_{1}-U_{1}=\mathscr{S}\{ & -\frac{8}{\pi^{3}}\left[\left(t-i \frac{\pi}{4}\right)^{3}+\frac{3}{2}\left(t-i \frac{\pi}{4}\right)^{2}(1+i) \zeta\right] \\
& -B\left[\left(t-i \frac{\pi}{4}\right)^{2}+\left(t-i \frac{\pi}{4}\right)(1+i) \zeta\right] \\
& \left.+b_{1} i\left(\frac{\pi}{4}-\frac{\zeta}{2}\right)+\frac{\zeta}{\pi}(3-i)-\frac{3 i}{2}\right\},
\end{aligned}
$$


and to order $\zeta^{3}$

$$
V_{1}=\mathscr{S}\left\{\begin{array}{l}
\zeta^{3} \\
\pi^{3}
\end{array}+B\left(\frac{\zeta^{2}}{4}-\frac{3}{8} \zeta^{3}\right)+b_{1}\left(\frac{\zeta}{2}-\frac{3}{8} \zeta^{2}+\frac{7}{24} \zeta^{3}\right)\right\} .
$$

Again we have

$$
\left[\frac{1-w^{1 / 2}}{1+w^{1 / 2}}\right]^{n}=\left(-\frac{\zeta}{2}\right)^{n}\left(1+\frac{\zeta}{2}\right)^{-n}
$$

so that

$$
\begin{aligned}
V_{2} & =\mathscr{F} \sum_{n=1}^{1} b_{n}\left[\frac{1-w^{1 / 2}}{1+w^{1 / 2}}\right]^{n}, \\
& =\mathscr{F}\left\{-b_{1}\left(\frac{\zeta}{2}-\frac{\zeta^{2}}{4}+\frac{\zeta^{3}}{8}\right)+b_{2}\left(\frac{\zeta^{2}}{4}-\frac{\zeta^{3}}{4}\right)-b_{3} \frac{\zeta^{3}}{8}\right\}, \text { approximately. }
\end{aligned}
$$

Also we note that

$$
\begin{aligned}
{\left[\begin{array}{c}
1+i w^{1 / 2} \\
1-i w^{1 / 2}
\end{array}\right]^{n} } & =\left[\begin{array}{c}
1+i+i \zeta \\
1-i-i \zeta
\end{array}\right]^{n} \\
& =i^{n}\left[1+n i \zeta+O\left(\zeta^{2}\right)\right],
\end{aligned}
$$

and so

$$
\begin{aligned}
U_{2} & =\mathscr{Y} \sum_{n=1}^{4} a_{n}\left[\frac{1+i w^{1 / 2}}{1-i w^{1 / 2}}\right]^{n} \\
& =\mathscr{Y}\left\{a_{1} i(1+i \zeta)-a_{2}(1+2 i \zeta)-a_{3} i(1+3 i \zeta)+a_{4}(1+4 i \zeta)\right\}, \quad \text { approximately. }
\end{aligned}
$$

Therefore to order $\zeta^{3}$, we have

$$
\begin{aligned}
& \psi^{\prime}=1+2 y^{\prime}+y^{\prime} \mathscr{Y}\left\{-\frac{8}{\pi^{3}}\left[\left(t-i \frac{\pi}{4}\right)^{3}+{ }_{2}^{3}\left(t-i \frac{\pi}{4}\right)^{2}(1+i) \zeta\right]\right. \\
& -B\left[\left(t-i \frac{\pi}{4}\right)^{2}+\left(t-i \frac{\pi}{4}\right)(1+i) \zeta\right]+b i\left(\frac{\pi}{4}-\frac{\zeta}{2}\right)+\frac{\zeta}{\pi}(3-i)-\frac{3}{2} i-b_{1} \frac{\zeta}{3} \\
& \left.-a_{1} i(1+i \zeta)+a_{2}(1+2 i \zeta)+a_{3} i(1+3 i \zeta)-a_{4}(1+4 i \zeta)\right\} \\
& +\mathscr{F}\left\{\begin{array}{l}
\zeta^{3} \\
\pi
\end{array}+B\left(\frac{\zeta^{2}}{4}-\frac{3}{8} \zeta^{3}\right)+b_{1}\left(\frac{\zeta}{2}-\frac{3}{8} \zeta^{2}+\frac{7}{24} \zeta^{3}\right)\right. \\
& \left.+b_{1}\left(-\frac{\zeta}{2}+\frac{\zeta^{2}}{4}-\frac{\zeta^{3}}{8}\right)+b_{2}\left(\frac{\zeta^{2}}{4}-\frac{\zeta^{3}}{4}\right)-b_{3} \frac{\zeta^{3}}{8}\right\} .
\end{aligned}
$$


Now from equation (33),

$$
\mathscr{F} \zeta^{2}=\frac{1}{2} \pi y^{\prime}+\mathscr{Y} \zeta_{3}^{3},
$$

so that subsituting for $\mathscr{Y} \zeta^{2}$ in the last equation, we get

$$
\begin{aligned}
\psi=1 & +y\left(-\frac{3}{8}+6 \frac{t^{2}}{\pi^{2}}+B \frac{\pi}{2}\left(t+\frac{1}{4}\right)+\frac{3 \pi}{16} b_{1}+\frac{\pi}{8} b_{2}-a_{1}+a_{3}\right) \\
& +y^{\prime}\left(\frac{15}{4 \pi}-\frac{12}{\pi^{3}}\left(t^{2}+t \frac{\pi}{2}\right)-B\left(t+\frac{\pi}{4}\right)-\frac{b_{1}}{2}+a_{1}-3 a_{3}\right] \cdot \zeta \\
& +y\left[-\frac{12}{\pi^{3}}\left(t^{2}-\frac{\pi}{2} t\right)-\frac{1}{4 \pi}-B\left(t-\frac{\pi}{4}\right)+2 a_{2}-4 a_{1}-\frac{b_{1}}{2}\right] . \zeta \zeta \\
+ & {\left[\frac{1}{\pi^{3}}-\frac{7}{24} B+\frac{b_{1}}{8}-\frac{b_{2}}{6}-\frac{b_{3}}{8}\right] . \zeta^{3} . }
\end{aligned}
$$

It is easily seen from equation (24) that the coefficient of $y$ in the above equation for $\psi^{\prime}$ is zero. A little calculation shows that the coefficients of $y^{\prime}, \zeta, \quad y^{\prime} \mathscr{H}^{\prime} i \zeta, \mathscr{I}^{3} \zeta^{3}$ respectively, are given by

$$
C_{1}=0.68720, \quad C_{2}=-0.66384, \quad C_{3}=0.28182 \text {. }
$$

So $\psi$ is now of the form

$$
\psi=1+y^{\prime} \mathscr{S}\left(C_{1}+i C_{2}\right) \zeta+C_{3} \mathscr{S} \zeta^{3} .
$$

Now set

$$
z=i+z^{\prime}=i+r^{\prime}\left(\cos \theta^{\prime}+i \sin \theta^{\prime}\right),
$$

so that $r^{\prime}, \theta^{\prime}$ are then polar coordinates with $C$ as pole, where we assume $0 \leqq \theta^{\prime} \leqq 2 \pi$. From equation (33) we have as a first approximation

$$
\zeta=\left[\begin{array}{c}
\pi r^{\prime} \\
2
\end{array}\right]^{1 / 2}\left(\cos \begin{array}{l}
\theta^{\prime} \\
2
\end{array}+i \sin \begin{array}{c}
\theta^{\prime} \\
2
\end{array}\right)
$$

and so in terms of these polar coordinates

$$
\psi=1+\left(\frac{\pi}{2}\right)^{1 / 2}\left(r^{\prime}\right)^{3 / 2}\left[\left(\frac{\pi}{2} C_{3}+\frac{C_{2}}{2}\right) \sin \frac{3 \theta^{\prime}}{2}+\frac{C_{2}}{2} \sin { }_{2}^{\theta^{\prime}}+2 C_{1} \sin _{2}{ }_{2}^{\theta^{\prime}} \cos \frac{\theta^{\prime}}{2}\right] .
$$

This must be a biharmonic function which is equal to 1 on the boundaries $\theta^{\prime}=0,2 \pi$ and whose normal derivative vanishes on these boundaries. In order to satisfy these conditions, we must have

$$
{ }_{2}^{3 \pi} C_{3}+2 C_{2}=0
$$


Subsituting the values previously obtained for $C_{2}, C_{3}$, we find that the error is only 0.0003 .

We also find that $\psi=1$ when $\theta^{\prime}=144^{\circ} 18^{\prime}$ and this gives the angle at which the stream line $\psi=1$ leaves the barrier.

The pressure $p$ is given by the equations of motion

$$
\begin{aligned}
& \partial p=-\mu \frac{\partial}{\partial y} \nabla^{2}{ }^{\prime}, \\
& \partial x=\mu \frac{\partial}{\partial x} \nabla^{2} \psi^{\prime} .
\end{aligned}
$$

where $\mu$ is the coefficient of viscosity of the fluid. That is to say, $p$ is the function conjugate to $\mu \nabla^{2} \psi$.

Remembering that $U$ and $V$ are harmonic, we have

$$
\nabla^{2} \psi=2+2\left(\frac{\partial V}{\partial y}-\frac{\partial U}{\partial y}\right)
$$

so that

$$
\partial y=2 \mu \frac{\partial^{2}}{\partial x \partial y}(V-U)
$$

and

$$
\partial p=-2 \mu \frac{\partial^{2}}{\partial y^{2}}(V-U)=2 \mu \frac{\partial^{2}}{\partial x^{2}}(V-U) .
$$

Therefore, apart from a constant,

$$
p=2 \mu_{\partial x}^{\partial}(V-U) .
$$

If $V-U=\mathscr{F} f(w)$, then we have

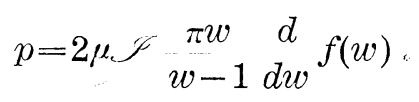

and using the expressions for $V$ and $U$, we obtain

$$
\begin{aligned}
& \frac{p}{2 \mu \pi}=\mathscr{F}_{2\left(1+w^{1 / 2}\right)(w-1)}^{1}\left\{3 A\left[\log \frac{2 w^{1 / 2}}{1+w^{1 / 2}}\right]^{2}+2 B\left[\log \begin{array}{c}
2 w^{1 / 2} \\
1+w^{1 / 2}
\end{array}\right]+G\right\} \\
& -\mathcal{F}_{2\left(i+w^{1 / 2}\right)(w-1)}^{i}\left\{3 A\left[\log \begin{array}{c}
2 w^{1 / 2} \\
i+w^{1 / 2}
\end{array}\right]^{2}+2 B\left[\log \frac{2 w^{1 / 2}}{i+w^{1 / 2}}\right]+C\right\} \\
& -\mathscr{S} \underset{w-1}{D}-\mathscr{F} \sum_{n=1} \frac{n b_{n} w^{1 / 2}}{\left(1+w^{1 / 2}\right)^{2}(w-1)}\left[\begin{array}{l}
1-w^{1 / 2} \\
1+w^{1 / 2}
\end{array}\right]^{n-1}
\end{aligned}
$$

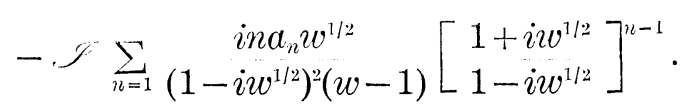


We will now calculate the pressure along the curve $u=0$, which inside the channel approximates to the central line.

Now when $w=r e^{i \pi / 2}$ then $w^{1 / 2}=\frac{r^{1 / 2}}{\sqrt{2}}(1+i)=\rho(1+i)$ say, and $w=2 i \rho^{2}$.

The expression for the pressure $p$ can be simplified as follows.

Let

$$
\log \frac{2 w^{1 / 2}}{1+w^{1 / 2}}=\lambda+i \gamma, \quad \text { say }
$$

then

$$
\lambda+i \gamma=\log 2+\log \rho+\log (1+i)-\log (1+\rho+i p)
$$

so that

$$
\lambda=\frac{1}{2} \log \frac{8 \rho^{2}}{1+2 \rho+2 p^{2}}, \quad \gamma=\tan ^{-1} \begin{gathered}
1 \\
1+2 \rho
\end{gathered} .
$$

Similarly we have

$$
\log \frac{2 w^{1 / 2}}{i+w^{1 / 2}}=\lambda-i \gamma
$$

Again we see that

$$
\begin{gathered}
\frac{1}{\left(1+w^{1 / 2}\right)}=\frac{1+\rho-i \rho}{1+2 \rho+2 \rho^{2}}, \\
i \quad=\frac{1+\rho+i \rho}{1+2 \rho+2 \rho^{2}}, \\
i+w^{1 / 2} \\
-\frac{w^{1 / 2}}{\left(1+w^{1 / 2}\right)^{2}}\left[\begin{array}{c}
1-w^{1 / 2} \\
\left.1+w^{1 / 2}\right]^{n-1}
\end{array}=-\rho(1+i) \frac{\left(1+2 i \rho^{2}\right)}{\left(1+4 \rho^{1}\right)}\left[\frac{1-2 \rho^{2}-2 i \rho}{1+2 \rho^{2}+2 \rho}\right]^{n},\right. \\
=\varepsilon_{n}+i \delta_{n} \quad \text { say, }
\end{gathered}
$$

and

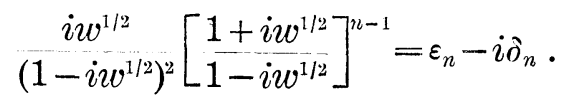

Therefore terms of the type

$$
\frac{1}{1+w^{1 / 2}}\left[\log \frac{2 w^{1 / 2}}{1+w^{1 / 2}}\right]-\frac{i}{i+w^{1 / 2}}\left[\log \frac{2 w^{1 / 2}}{i+w^{1 / 2}}\right]
$$

are the difference of two conjugate quantities, and putting in the values of $A, C, D$, and $G$, the expression for $p$ is 
(34)

$$
\begin{aligned}
& \frac{p}{2 \mu \pi}=\left\{\mathscr { \mathscr { S } } \left[\begin{array}{c}
24 \\
\pi^{3}\left(1+w^{1 / 2}\right)
\end{array}\left(\log \frac{2 w^{1 / 2}}{\left(1+w^{1 / 2}\right)}\right)^{2}+\frac{2 B}{\left(1+w^{1 / 2}\right)} \log \frac{2 w^{1 / 2}}{1+w^{1 / 2}}\right.\right. \\
& \left.\left.+\begin{array}{c}
b_{1} \\
1+w^{1 / 2}
\end{array}\right]\right\} \times\left\{\mathscr{S} \frac{i}{w-1}\right\}+\mathscr{S} \frac{1}{(w-1)}\left[\frac{2}{\pi}-\frac{i}{\pi\left(i+w^{1 / 2}\right)}\right] \\
& +\left\{\mathscr{S}_{w-1}^{1} \cdot\left[\sum_{n=1}^{\infty} n \varepsilon_{n}\left(b_{n}-a_{n}\right)+i \sum_{n=1}^{\infty} n \delta_{n}\left(b_{n}+a_{n}\right)\right]\right\}
\end{aligned}
$$

An arbitrary constant could be added to this expression, but as the formula stands $p$ is zero when $|w|$ is large, and so it denotes the excess of pressure at any point above that at a distant point outside the channel.

It can now be verified that at a distance from 0 outside the barriers, the pressure is approximately a linear function of $x$.

We have the exact relation between $z$ and $\rho$

$$
\pi z=2 i \rho^{2}-\log 2 i \rho^{2}+i \pi-1
$$

giving

$$
\pi x=-\log 2-2 \log \rho-1 .
$$

When $|w|$ is small the pressure is given approximately by

$$
\frac{p}{2 \mu \pi}=-\frac{24}{\pi^{3}} \cdot \frac{\pi}{2}\left[\begin{array}{l}
3 \\
2
\end{array} \log 2+\log \rho\right]-B_{2}^{\pi} .
$$

and substituting for $\log \rho$ in terms of $x$ we get

$$
p=12 \mu x+\frac{12}{\pi} \mu(1-2 \log 2)-B \pi^{2} \mu,
$$

that is to say

$$
p=12 \mu x+3.66 \mu .
$$

It has been shown that in this region $\psi^{\prime}=3 y^{2}-2 y^{3}$, and it can be seen that $12 \ell^{\prime}$ is indeed the pressure gradient required to maintain the motion given by this stream function in an infinite channel. This fact provides a check on the calculated value for $p$ for large $x$.

Figure 3 shows the graph of $p / \mu$ potted against $x$, as calculated from formula (34), with $n=4$.

It has been assumed in our work that the distance between the barriers is unity, and that $y^{2}$ is the stream function of the undisturbed motion, giving 2 as the undisturbed velocity at a unit distance from the infinite boundary. If the distance between the barriers is $a$, and $q$ is the undisturbed velocity at a distance $a$ from the infinite boundary, then we have to apply a factor $q / 2 \alpha$ to our results. 


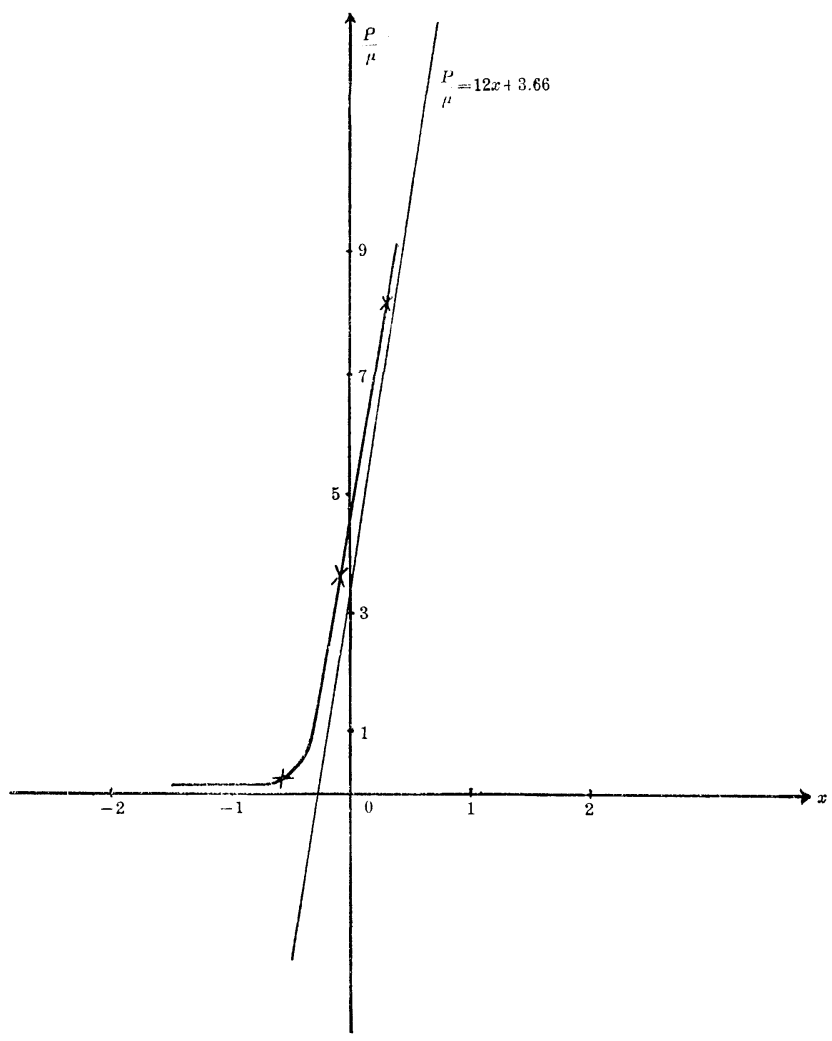

Fig. 3.

Since the equations of slow steady flow of a viscous incompressible fluid are the same as those of an equilibrium state of an incompressible elastic solid, the previous results can be immediately used for the solution to a certain two-dimensional elastic problem, if one simply replaces velocity and coefficient of viscosity by displacement and shear modulus. In the fluid problem there is ideally no velocity along the boundaries, so that in the corresponding elastic problem the boundaries could be free from tractions. A method of obtaining solutions to a large number of two-dimensional viscous flow problems from known elastic fields has been discussed by Goodier [2], who pointed out that an elastic stressfunction, being biharmonic, could be regarded as the stream-function of a viscous flow, and that the boundary conditions on the elastic stresses could be related to those on the fluid velocity.

\section{REFERENCES}

1. W. R. Dean, On the slow viscous motion near a half-pitot tube, Proc. Cambridge Philos. Soc., 48 (1952), 149.

2. J. H. Goodier, An analogy between the slow motions of a viscous fluid in two dimensions and systems of plane stress, Philos. Mag., 17 (1934), 554.and 800. 



\section{PACIFIC JOURNAL OF MATHEMATICS}

EDITORS

\author{
H. L. Royden \\ Stanford University \\ Stanford, California \\ E. Hewite \\ University of Washington \\ Seattle 5 , Washington
}

\author{
R. P. Dilworth \\ California Institute of Technology \\ Pasadena 4, California
}

\author{
A. Horn* \\ University of California \\ Los Angeles 24, California
}

\section{ASSOCIATE EDITORS}
E. F. BECKENBACH
M. HALL
M. S. KNEBELMAN
J. J. STOKER
C. E. BURGESS
P. R. HALMOS
I. NIVEN
V. GANAPATHY IYER
T. G. OSTROM
G. SZEKERES
H. BUSEMANN
R. D. JAMES
M. M. SCHIFFER
F. WOLF
H. FEDERER

\section{SPONSORS}

\author{
UNIVERSITY OF BRITISH COLUMBIA \\ CALIFORNIA INSTITUTE OF TECHNOLOGY \\ UNIVERSITY OF CALIFORNIA \\ CALIFORNIA RESEARCH CORPORATION \\ MONTANA STATE UNIVERSITY \\ UNIVERSITY OF NEVADA \\ OREGON STATE COLLEGE \\ UNIVERSITY OF OREGON \\ UNIVERSITY OF SOUTHERN CALIFORNIA
}

\author{
STANFORD UNIVERSITY \\ UNIVERSITY OF UTAH \\ WASHINGTON STATE COLLEGE \\ UNIVERSITY OF WASHINGTON \\ AMERICAN MATHEMATICAL SOCIETY \\ HUGHES AIRCRAFT COMPANY
}

Mathematical papers intended for publication in the Pacific Journal of Mathematics should be typewritten (double spaced), and the author should keep a complete copy. Manuscripts may be sent to any of the editors. Manuscripts intended for the outgoing editors should be sent to their successors. All other communications to the editors should be addressed to the managing editor, Alfred Horn at the University of California, Los Angeles 24, California.

50 reprints of each article are furnished free of charge; additional copies may be obtained at cost in multiples of 50 .

The Pacific Journal of Mathematics is published quarterly, in March, June, September, and December. The price per volume (4 numbers) is $\$ 12.00$; single issues, $\$ 3.50$. Back numbers are available. Special price to individual faculty members of supporting institutions and to individual members of the American Mathematical Society: $\$ 4.00$ per volume; single issues, $\$ 1.25$.

Subscriptions, orders for back numbers, and changes of address should be sent to Pacific Journal of Mathematics, c/o University of California Press, Berkeley 4, California.

Printed at Kokusai Bunken Insatsusha (International Academic Printing Co., Ltd.), No. 10, 1-chome, Fujimi-cho, Chiyoda-ku, Tokyo, Japan.

* During the absence of E. G. Straus.

PUBLISHED BY PACIFIC JOURNAL OF MATHEMATICS, A NON-PROFIT CORPORATION COPYRIGHT 1956 BY PACIFIC JOURNAL OF MATHEMATICS 


\section{Pacific Journal of Mathematics}

\section{Vol. 6, No. $2 \quad$ December, 1956}

Louis Auslander, Remark on the use of forms in variational calculations .......................................... 209

Hubert Spence Butts, Jr. and Henry B. Mann, Corresponding residue systems in algebraic number fields ........................ 211

L. Carlitz and John Herbert Hodges, Distribution of matrices in a finite field............................................

Paul Civin and Bertram Yood, Invariant functionals ............... 231

David James Dickinson, Henry Pollak and G. H. Wannier, On a class of polynomials orthogonal over a denumerable set .................

Bernard Friedman and Luna Mishoe, Eigenfunction expansions associated with a non-self-adjoint differential equation ....................

Luna Mishoe and G. C. Ford, On the uniform convergence of a certain eigenfunction series .............................. 271

John W. Green, Mean values of harmonic functions on homothetic curves...........................................

Charles John August Halberg, Jr. and Angus E. Taylor, On the spectra of linked operators .....................................

Chuan Chih Hsiung, Some integral formulas for closed hypersurfaces in Riemannian space ................................... 291

Norman D. Lane, Differentiable points of arcs in conformal n-space 301

Louis F. McAuley, A relation between perfect separability, completeness, and normality in semi-metric spaces

G. Power and D. L. Scott-Hutton, The slow shearing motion of a liquid past a semi-infinite plane .............................

A. C. Schaeffer, Entire functions

Edward Silverman, An intrinsic inequality for Lebesgue area...

Choy-Tak Taam, Asymptotic relations between systems of differential equations.

Ti Yen, Quotient algebra of a finite $A W^{*}$-algebra ... 